\title{
Treatment Return Date
}

National Cancer Institute

\section{Source}

National Cancer Institute. Treatment Return Date. NCI Thesaurus. Code C82514.

The date that the study treatment was returned. 